\title{
DESARROLLO URBANO Y DESIGUALDAD EN BAHIA BLANCA
}

Stella Maris Pérez*

\section{Resumen}

\begin{abstract}
El objetivo del trabajo es el describir la problemática de la desigualdad y su relación con la situación urbana en la ciudad de Bahía Blanca, especialmente en los aspectos de la vivienda y en diferencias no relacionadas directamente con la situación económica. Se intenta comprender los condicionamientos que el desarrollo urbano impone a los habitantes de la ciudad, y es la intención de este capítulo hacer uso de los mismos, especialmente de aquellos vertidos en el texto "City Differences", en función de poder presentar diferencias (desigualdades) "no económicas" al interior de la ciudad. Así la segmentación del mercado de trabajo, la recepción de migración interna y de países limítrofes, las crisis económicas a nivel nacional, el cambio de las principales actividades productivas (otrora orientadas al agro y actualmente al desarrollo de la industria petroquímica) y sus vinculaciones con la división social del espacio, son analizadas como algunos de los factores que inciden en la complejización de las desigualdades (económicas, culturales, religiosas, etc.) al interior de la estructura social. Esquemáticamente el trabajo se compone de una caracterización a nivel macrosocial del impacto ocasionado por el desarrollo urbano en Bahía Blanca sobre la base de trabajos realizados por el Gobierno Municipal y el Departamento de Geografía de la Universidad Nacional del Sur, describiendo el escenario de la ciudad en las últimas décadas. Las modificaciones de su estructura social serán luego presentadas en base a los indicadores clásicos (ocupación, educación, etc.) para detenerse en aquellas diferencias que los entrevistados perciben como las más importantes. Estos últimos datos provienen de grupos focalizados y permiten, conjuntamente a otras fuentes de datos estadísticos, situar el escenario actual de la pobreza.
\end{abstract}

Clasificación JEL: I31 - R2

Palabras clave: desarrollo urbano - vivienda - desigualdad

Abstract

The aim of this paper is to describe the problem of inequality and its relationship with the urban situation in Bahía Blanca City, especially in those aspects that have to do with

"Departamento de Economía, Universidad Nacional del Sur, smperez@criba.edu.ar. 
housing conditions and facilities and in certain differences that are not related to the economic situation. In order to understand how urban development influences in people behaviour, this work introduces "City Differences" key - words to express non-material differences among the citizens. Labour market broken structure, internal migration, as well as that coming from bordering nations, the national economical crisis, fundamental changes in the most important productive activities (those that were formerly based on agriculture and have been turned towards the industrial development of petrochemistry) and its associations with the social space division, are analized as some of the reasons that determine complexity in inequality (in the economical and cultural aspects) in social structure. This outline refers, first of all, to the macro-social aspects that urban development impact had on Bahía Blanca and it is based on a research work carried out by the Local Government and the Geography Department of The National University of the South (UNS), describing the development of the city during the last decades. Furthermore, it will explain how the social structure has been modified taking into account the most classical indicators, such as occupation, education, etc., especially those which are more important in people's opinion. The data is the product of survey made on specific groups of people, as well as statistics data, and gives a picture of the situation of poverty.

JEL Classification: I31 - R2

Keywords: urban development - household - inequality

\section{INTRODUCCION}

La descripción y explicación de problemáticas vinculadas a la exclusión, marginalidad y pobreza no constituye un tema nuevo en las ciencias sociales. La especificidad de este trabajo es la presentación de material empírico que relaciona desigualdades materiales (o económicas) con otras simbólicas (culturales, religiosas, etc.) en el marco del desarrollo urbano de la ciudad de Bahía Blanca.

Cabe advertir que no es objetivo de este trabajo, elaborar un mapa que vincule la distribución espacial con la social. Se pretende en cambio, organizar una serie de información proveniente de distintas fuentes en base a las categorías de análisis antes señaladas (desarrollo urbano, estructura social, asentamientos, desigualdad) contextualizando y enriqueciendo el conocimiento sobre las nuevas formas de pobreza que aparecen en la ciudad. En relación al último punto, también es importante reflexionar más allá de los criterios relacionados al ingreso y ampliar el análisis a otros aspectos. 
Fundada en 1828 en las márgenes de la bahía, la ciudad de Bahía Blanca, surgió como "Fortaleza Protectora Argentina", siendo su crecimiento lento hasta fines del siglo XIX. Sin embargo desde mediados del mismo, el desarrollo de la ganadería y el avance del modelo agroexportador, hicieron que, al momento del Censo de 1869, su fisonomía hubiese adoptado la forma de un "gran poblado" y se constituyese como uno de los centros más importantes del sur argentino.

Para el 2001 la ciudad contaba con una fisonomía muy particular, con una población de aproximadamente 400.000 habitantes, con gran vinculación económica con el desarrollo agropecuario (aunque el nivel de urbanización del partido es del $98.5 \%$ ), y con una serie de industrias alimenticias fundadas durante la mitad del siglo pasado, así como también con un importante Polo Petroquímico y la existencia de un puerto de aguas profundas.

Estas modificaciones estructurales de la ciudad impactan de tal manera, que sus efectos se perciben, no sólo a nivel ocupacional como se evidenció en las altas tasas de desempleo de la década del '90 o en la precarización de la ocupación, sino que repercuten en todo el tejido social, como sucede con las políticas sociales organizadas bajo la forma de planes "con contraprestación", que también alimentan estas actividades no calificadas, mal remuneradas y muy diferentes de lo que se considera "trabajo asalariado". Este proceso se relaciona directamente con importantes cambios en la estructura social y en la expansión y heterogeinización de las condiciones de pobreza.

Es preciso detenerse en este último concepto, pues su conceptualización y relación con otras variables es fundamental para la comprensión de la dinámica que se pretende analizar. Aquí se adopta la perspectiva de entender a la pobreza como un problema directamente vinculado con la estructura social, donde amplios sectores de la población sufren situaciones de movilidad descendente y deterioro de sus condiciones de vida, hasta el punto de que sus capacidades para el logro del bienestar se ven seriamente afectadas 1.

Es verdad que los dos criterios utilizados en las fuentes de datos sistematizadas en la Argentina (insatisfacción de las necesidades básicas y línea de

\footnotetext{
${ }^{1}$ Esta visión de la pobreza entendida más allá de la distribución de ingresos y en una perspectiva relacional remite al pensamiento de Amartya Sen. Es importante señalar que no es el único autor que plantea este enfoque, pero su mención se realiza reconociéndolo como la vertiente que se ha atendido preferentemente para este concepto en la formulación de este trabajo.
} 
pobreza), remite a definiciones más limitadas, pero es importante indicar estas cuestiones y asumir que la problemática de la pobreza obliga a revisar los instrumentos de medición y agregar otros aspectos no contemplados en dichos mecanismos .

No es objetivo de este trabajo plantear nuevos criterios de medición de la pobreza, pero esta digresión permite (como se verá más adelante) remitir el análisis al estudio de ciertas condiciones de vida y desigualdades no económicas que redundan también en diferencias espaciales. Esto por supuesto, sin descuidar las desigualdades económicas características de las mediciones clásicas de la pobreza.

Las transformaciones globales definen directamente transformaciones en el espacio urbano. Algunos de estos impactos son de carácter económico (como los ocupacionales que se mencionaron antes) y otros culturales o simbólicos, pero todos ellos confluyen en cerrar una espiral dialéctica que muestra la influencia de estos efectos globales en todos los ámbitos sociales y a su vez los retroalimentan. Así, en primer lugar, se intentará caracterizar el proceso global de transformación del espacio urbano para el caso de Bahía Blanca, para luego detenerse en la caracterización de la vivienda y otras formas de diferenciación.

\section{LAS TRANSFORMACIONES DEL ESPACIO URBANO EN BAHIA BLANCA}

El proceso de globalización tiene sus consecuencias también en la conformación del nuevo espacio urbano. Complejo por definición, determina entre otros efectos, la fragmentación de las ciudades.

Mientras que en América Latina la planificación urbana no tiende a reproducir la división racial del espacio, en Europa y Estados Unidos los flujos migratorios transforman la lucha de clases en una lucha étnica con importante impacto en la configuración de las ciudades. En cambio para todos los casos, puede observarse la aparición de la "ciudad dual". Dicho proceso implica una tendencia a la polarización extrema y al aumento de las diferencias materiales y simbólicas. A esta ampliación de la brecha, deben sumarse las diversas distinciones que se verifican al interior de las clases.

Este proceso de heterogeinización no debe ser entendido sólo en el plano económico: las diferencias culturales, identidarias, comunicacionales, 
valorativas y de poder, se multiplican, crecen en importancia respecto a las diferencias económicas, y posibilita el análisis de la exclusión en forma más exhaustiva, y la inclusión de nuevos motivos como fuentes posibles de asociación social o política.

Estas múltiples diferencias sociales, se traducen en nuevas diferencias espaciales, adquiriendo el problema de la segregación nuevas dimensiones. No sólo se asiste a la segregación pasiva- la sufrida por ejemplo por los pobres que son rechazados por distintos grupos, y/o sin acceso al suelo y la vivienda- y la segregación activa, característica de los grupos sociales de mayores recursos, que deciden vivir separados, a veces por temor a la violencia urbana, y otras veces en la búsqueda de mejor calidad de vida (Schteingart, 2001).

Estos enfoques de la división del espacio social, relacionada con la perspectiva teórica de las nuevas formas de la pobreza, son característicos de la década del ' 80 y se remiten por lo general a los estudios de casos, pero como ya se planteó, coinciden en una serie de características observadas para todas las ciudades: la crisis del espacio público y las nuevas fragmentaciones territoriales son algunos ejemplos.

Sin embargo, este trabajo rescata el tema de la pobreza vinculándolo con la problemática más general de las clases sociales y la estratificación social como referentes de la estructura socio-espacial, como fue característico en las décadas del '60 y del '70 (Schteingart, 2001). Así, se entiende a la fragmentación del espacio urbano como uno de los principales condicionamientos negativos para la integración social. Si bien antes la ciudad se conceptualizaba como lugar propicio para las interacciones entre distintos grupos sociales, actualmente la multiplicidad de espacios e intercambios parece haber disminuido (Fundación BAPRO, 2005). Esto se debe, entre otros factores, a que el desarrollo de las grandes ciudades latinoamericanas se ha dado bajo el modelo del Estado de Bienestar. Desde la década del '90 se relega este modelo y los cambios profundos debidos a la intervención estatal han generado que sean preferentemente los mecanismos del mercado quienes conformen la situación del espacio urbano. Estos mecanismos promueven la privatización de espacios públicos (aparición masiva de centros comerciales) como "necesaria" por razones de exclusividad y seguridad, acentuándose la fragmentación de la unidad urbana y su reemplazo por territorios marcadamente identitarios (Prevot- Schapira, 2001).

La situación en Bahía Blanca, como en la mayoría de las ciudades medianas y pequeñas de la Argentina, no es tan extrema (existen espacios públicos donde 
interactúan individuos de diversas clases sociales, es menor la segregación espacial y la polarización entre las mejores y peores viviendas que en ciudades grandes, etc.), aunque las modificaciones en el uso del espacio urbano han sido importantes en los últimos años, y se relacionan con modificaciones estructurales de otro orden. En la ciudad, durante la década del '90, se registraron altísimas tasas de desempleo y sub-empleo: 31.4\% para mayo de 1996 (EPH/ INDEC-Informe 1997Vol. 1 №12). Algunas de las principales causas se atribuyen a la racionalización de las empresas de servicios y a la reconversión del Polo Petroquímico y las instituciones bancarias. También es importante considerar el crecimiento de la población económicamente activa debido a la inmigración de trabajadores provenientes de países limítrofes, el crecimiento explosivo del cuentapropismo y el fracaso de gran cantidad de proyectos financiados con ingresos provenientes de retiros voluntarios, entre otros.

El impacto de dichos sucesos se hizo sentir en toda la población, pero afectó en mayor proporción a los sectores de pocos recursos. De las múltiples consecuencias de este proceso, interesa en este punto, focalizar la atención en un problema que afecta a la división del espacio: el crecimiento físico de los asentamientos marginales existentes, la incorporación de nuevos en otras áreas urbanas y el consecuente deterioro de las condiciones habitacionales (Formiga y Garriz, 1998).

La población de la ciudad ha mantenido un crecimiento sostenido en los últimos años. Durante las décadas del '70 y del ' 80 , se produjeron dos procesos importantes en su desarrollo y cambio en su configuración económica y espacial. La ciudad antiguamente orientada al agro, cambiaba su perfil a partir de sentar las bases para el desarrollo industrial químico y petroquímico, con la radicación de la planta Petroquímica Bahía Blanca (1981) constituida como sociedad mixta (51\% del Estado y $49 \%$ capitales privados); y con el avance energético implícito en la construcción del Gasoducto Neuba 2 y de la Central Termoeléctrica Luis Piedrabuena (1989).

Estos acontecimientos fueron acompañado son con un importante caudal de población migrante proveniente de zonas rurales, especialmente el sudoeste de la provincia de Buenos Aires del Alto Valle del Río Negro2, expulsada por las distintas crisis que afectaron al sector agrícola. Dicho crecimiento demográfico, incentivado en la década del '90 por la dinámica económica implementada desde 1995 con la

\footnotetext{
${ }^{2}$ Según el Censo 91, el 36\% de su población había nacido fuera de la ciudad.
} 
privatización y crecimiento de la petroquímica al ser adquirida por Dow Chemical, YPF e Itochu, la descentralización del Complejo Portuario, y el proyecto de Zona Franca; impulsó el crecimiento del casco urbano. En forma contraria en el marco de la globalización de los años '90, la ciudad fue adquiriendo una nueva proyección. La descentralización del espacio portuario, junto al procesos de inversiones, son elementos que caracterizan el presente panorama económico de la ciudad y que interactúan a favor del posicionamientos de Bahía Blanca en el horizonte regional extra- nacional (Zingoni, 1998).

La ciudad presenta un micro y un macro centro dentro de un precinto constituido por los arroyos y las vías férreas, con densidades habitacionales que varían entre los 60 hab/ha y los 300-500hab/ha en los alrededores de la Plaza central, "donde se verifica la totalidad de los equipamientos y de la infraestructura de servicios" (Zingoni, 1998). Además de concentrar la mayor cantidad de edificios, el centro se caracteriza por combinar el uso comercial con el residencial, siendo importante el atractivo y concentración que ejerce para el desarrollo del trabajo informal. Más allá de este precinto, la ciudad se extiende hacia el Este/ Noreste y hacia el SE/SO (donde forma un anillo costero que limita al puerto), predominando las casas de una o dos plantas, y sólo en forma puntual, edificios en altura.

Dentro del ejido urbano, los asentamientos marginales 4 se localizan en dos focos importantes situados en el oeste y en el sur. El primero, con siete de ello, concentra al $40 \%$ de la población residente en asentamientos, en una zona de marginalidad que pesa en su integración con las áreas circundantes, no obstante que forma parte de la trama urbana. El segundo, con nueve núcleos, sigue el trazado del arroyo Napostá Norte, lo que induce a la continuidad espacial entre las villas en un continuo que comienza a sólo 13 cuadras del centro comercial y administrativo de la ciudad, y se prolonga hasta inmediaciones de la zona portuaria de Ing. White, albergando al $21 \%$ de los habitantes en forma asentamientos. En ellos se acentúan las condiciones de marginalidad observadas en los de la zona oeste (Formiga y Garriz, 1998).

\footnotetext{
${ }^{3}$ El caso de otras ciudades argentinas como Buenos Aires, presenta un uso intensivo institucional y comercial del microcentro sin coexistencia con el uso habitacional. $4 \mathrm{El}$ trabajo que se ha tomado como regencia define como primer criterio de marginalidad a la "ilegalidad de la tenencia de la tierra", utilizando para la identificación, localización y caracterización de estas unidades de estudio a información proveniente del Departamento de Catastro de la Municipalidad de Bahía Blanca. (Formiga y Garriz, 1998: 104-105)
} 
El resto de los asentamientos se distribuye de manera irregular por el resto de la ciudad, siendo los dos más importantes los denominados Villa Miramar y Stella Maris, con el 15 y $8 \%$ del total de la población carenciada de la ciudad, ambos sobre el este de la ciudad.

El origen de estos asentamientos remite a la década del '60 y aún en los '70, detectándose la mayor intensidad en la formación de áreas marginales. La población se asentó donde las reglas del mercado lo permitían, conformándose un mosaico de llenos y vacíos que dio como resultado un patrón de suelo discontinuo con baja calidad ambiental por falta de equipamiento y de infraestructura básica de servicios, ocasionando una disminución en los valores de la tierra (Zingoni, 1998). La importancia de este fenómeno en la Argentina, se traduce en una periferia de baja calidad ambiental, desequilibrio y segregación socials.

Durante la década del '80, la reformulación del Plan de Desarrollo Urbano de Bahía Blanca de 1985 plantea una serie de erradicaciones y transferencias de la población a otros sectores. Las causas de la erradicación se encontraban en la necesidad de esas tierras para obras públicas: entubado del arroyo Napostá y parque Campaña al Desierto. Los barrios erradicados fueron trasladados a sectores periféricos de la ciudad, donde construyeron sus viviendas por sistemas cooperativos o de autoconstrucción con asesoramiento municipal (Formiga y Garriz, 1998). Sin embargo, la inaccesibilidad al centro desde estas zonas y los problemas de integración de los nuevos habitantes con los que ya residían allí, redundaron en la reproducción de las condiciones de precariedad del hábitat y de su calidad de vida (Formiga y Garriz, 1998).

Finalmente en los años '90 aparecen otros cuatro asentamientos. El último de ellos, Villa Roldán de 1995, presenta en la actualidad un sostenido crecimiento con manifiesta precariedad en su infraestructura por encontrarse en una zona inundable, entre las vías del ferrocarril y la ruta de acceso a los puertos. Otro asentamiento de la década de los '90 es Villa Sapito, que surge por la relocalización de familias que vivían en tierras del Club Golf Palihue (Formiga y Garriz, 1998). Aquí puede observarse que la erradicación no se realizó por una cuestión de desarrollo urbano, sino por ciertos intereses privados. El Palihue, barrio donde se ubica y da

\footnotetext{
${ }^{5}$ Ver para el caso de México los trabajos de Schteingardt y para Córdoba el de Luciano, Francisco: Regularización de asentamientos irregulares en Córdoba, en Azuela y Tomas. Para Buenos Aires: Torres, Horacio El mapa social de Buenos Aires (1940-1990), Facultad de Arquitectura, Diseño y Urbanismo-UBA.
} 
nombre al club, se encuentra separado del trazado urbano de la ciudad por las vías del ferrocarril, el arroyo Napostá, la avenida Sarmiento (el acceso más directo) y predios privados, como el golf. La erradicación del mencionado asentamiento no fue la única medida tendiente al aislamiento de esta zona residencial de sectores altos y medios altos: la ampliación del predio de la Universidad del Sur a partir del 2000 , también produjo una serie de manifestaciones y reclamos por parte de los vecinos ante la extensión del transporte público. Así, desde los 90, las políticas de erradicación y localización refuerzan la segregación y el aislamiento espacial.

El proceso de crecimiento del espacio urbano ocupado por estos asentamientos se verifica a través de la comparación de fotos aéreas correspondientes a los años 1990 y 1996 (Formiga y Garriz, 1998) donde se concluye que dicho crecimiento se expresa por una densificación del hábitat y por expansión de la superficie ocupada, aunque en cuanto su magnitud no se trate de un crecimiento muy importante, pero si de muy precaria condición.

La expansión de viviendas de nivel medio- alto y alto en la década del '90 y en los últimos cinco años, se dio en zonas alejadas del centro, y en algunos casos sub- urbanizadas (por ejemplo: Pago Chico, Patagonia Norte, Bosque Alto, Villa Belgrano, etc.), siguiendo un modelo poco común en Latinoamérica, aunque más difundido en Estados Unidos.

En diciembre de 1995, el entonces intendente municipal al momento de asumir su segundo mandato, presentó una propuesta innovadora al abordar la temática de la ciudad, convocando a la apertura y la participación de todos los sectores que conforman la comunidad de Bahía Blanca (Zingoni, 1998). Dicha "participación" se tradujo en la redacción de un Plan Estratégico entre distintos organismos, colegios profesionales, universidades, etc., orientando la planificación de la ciudad, tales como servicios, rutas de acceso, parquización, embellecimiento, atracciones turísticas. Este tipo de política orientada a la institucionalización de la participación, reemplaza al enfrentamiento del Estado con los distintos movimientos urbanos y podría clasificarse dentro de las formas de participación citadas por Schteingart y Duhau (2001) como más elaboradas (colaboración en planes de gobierno).

A partir del año 2004, con el cambio de gestión municipal, que implicó también el cambio en el partido político gobernante, se observa un giro en las políticas orientadas al desarrollo urbano. Si antes se orientaban a la planificación estratégica, ahora se la vincula directamente con las políticas de desarrollo social de los sectores más postergados. En este sentido, la política habitacional ocupa un 
lugar importante, y se promueve (entre otras acciones), la elaboración de un padrón de hogares con necesidades habitacionales.

A pesar de que el empadronamiento no fue exhaustivo, se observa que estos hogares viven en su gran mayoría en condiciones de pobreza relativa, pero que la calidad de las viviendas ocupadas en la actualidad es mejor que la observada en otras ciudades del país: ....se trata de una población joven, de hogares en expansión, cuyo nivel de ingreso les impide el acceso al crédito comercial, pero que no se encuentra viviendo en condiciones de pobreza extrema. En cuanto a las características habitacionales, ..., el indicador que arroja datos más pesimistas es el de hacinamiento, encontrándose en situación crítica 963 hogares (17.3\%)6 (Burstein y Pérez, 2004)

Otros estudios sobre la población total, como la Encuesta de Desarrollo Social de 1997 del SIEMPRO, contabiliza sólo el 2.8\% de viviendas deficientes, muy por debajo del $4.9 \%$ observado a nivel nacional. En cuanto a la ubicación de las mismas, el $79.4 \%$ se encuentra en un medio urbano no deficitario, apareciendo el resto de las mismas distribuidas en un $0.4 \%$ en villas de emergencia, el $6.6 \%$ en barrios con calles de tierra y el $13.6 \%$ en barrios con construcción precaria (Pérez, 2003) 7 .

Todos estos datos coinciden con lo observado por Torrado para las ciudades pequeñas y medianas: las condiciones de vida suelen ser mejores que en las grandes urbes, porque el acceso a la vivienda y otros servicios es más fácil, y porque la capacidad de constituir redes de ayuda social son mayores. (Torrado: 367 -377). Así, tanto el grado de hacinamiento, como la disponibilidad de agua dentro de la vivienda (indicadores habitacionales cuya potencia discriminatoria es mayor), presentan guarismos favorables para la amplia mayoría de la población: ....se puede concluir que el número de viviendas en condiciones precarias es más pequeño que en el nivel nacional. Es verdad que la calidad de dichas viviendas ha sido afectada negativamente en estos últimos cinco años debido a la caída de los ingresos, pero la vivienda medida a través de estos indicadores (agua y baño) es una medida estructural cuyas modificaciones son lentas en lo que respecta a la dimensión temporal del cambio (Pérez, 2003).

\footnotetext{
${ }^{6}$ La EPH de mayo del 2001, presenta un $3.1 \%$ de hacinamiento crítico para el conglomerado Bahía Blanca.

7Las categorías "villas de emergencia", "barrios con calles de tierra" y "barrios con construcción precaria" son excluyentes entre sí, pero todas remiten a déficit habitacional.
} 
En síntesis, los impactos del desarrollo urbano se han traducido preponderantemente en la ampliación de zonas habitadas (posibilitada a partir de grandes extensiones de tierra habitable y de la modernización de los caminos y accesos a la ciudad), y en la modificación del espacio urbano por la aparición de barrios cerrados y barrios parque, apertura de importantes centros comerciales y modernización de edificios públicos del centro. Sin embargo, como se observará más adelante con las cifras presentadas, la situación de los sectores sociales más bajos no muestra las mismas condiciones de precariedad observadas para el total nacional o para el área metropolitana de la ciudad de Buenos Aires.

La división social del espacio no ha sido estudiada en la ciudad de Bahía Blanca con la profundidad suficiente como para estipular si la misma muestra procesos de concentración y polarización en los últimos años. Sí, existen trabajos que muestran un importante crecimiento urbano y paralelamente un proceso de movilidad social descendente, pero sin vincular un proceso social al otro. Sin embargo, puede afirmarse que la división del espacio reproduce una estratificación social relativamente homogénea en los distintos indicadores, que empuja al uso de criterios simbólicos y subjetivos en la interacción y diferenciación de los individuos. El punto que sigue, profundiza el tema de la estructura de clases, para luego detenerse en las distinciones "no económicas" que surgen en la interacción.

\author{
II. DESIGUALDAD SOCIAL, DIVISION DEL ESPACIO \\ Y CONDICIONES HABITACIONALES EN BAHIA BLANCA
}

Las distintas fuentes de datos sistematizadas y disponibles a nivel nacional permiten el estudio de la desigualdad a través de una serie de indicadores e índices tales como ingreso, educación, ocupación, bienes y vivienda. En lo que se refiere al ingreso, se observa en Bahía Blanca un ingreso per cápita menor al promedio nacional (Cuadro 1), pero su distribución es más homogénea que en el resto del país y otros agregados (Cuadro 2). Esta forma de la distribución genera un acercamiento entre los distintos sectores que conforman la pirámide social apelándose a otros indicadores para determinar la clase social a la que pertenece una persona y distinguir un estrato de otro. 
Cuadro 1: Ingresos de la población bahiense y total del país, 1997 - 2001

\begin{tabular}{|l|c|}
\hline \multicolumn{1}{|c|}{ Ingreso Medio } & Conglomerado \\
\cline { 2 - 2 } & Total NacionalBahía Blanca \\
\hline Hogares (\$- 1997- EDS/ EPH) & 81136.7946 \\
Per cápita (\$- 1997- EDS/ EPH)) & 284308 \\
Hogares (\$- 2001- EPH) & 779935 \\
Per cápita (\$- 2001- EPH) & 256268 \\
\hline
\end{tabular}

Fuente: Encuesta de Desarrollo Social (1997) - SIEMPRO y EPH (2001) - INDEC. Procesamiento propio sobre datos de Bahía Blanca.

Cuadro 2: Ingresos de la población bahiense, total del país, región pampeana y provincia de Buenos Aires

\begin{tabular}{|c|c|c|c|c|c|}
\hline \multirow[t]{2}{*}{ Conglomerado } & \multicolumn{5}{|c|}{ Quintiles de ingreso per cápita del hogar } \\
\hline & 1 & 243 & & & 5 \\
\hline $\begin{array}{l}\text { Bahía Blanca } \\
\text { Provincia de Buenos Aires } \\
\text { Región Pampeana } \\
\text { Total nacional }\end{array}$ & $\begin{array}{l}20.1 \% \\
12 \% \\
9.1 \% \\
9.1 \%\end{array}$ & $\begin{array}{l}19.8 \% 20.7 \\
14.5 \% 21.6 \\
16.1 \% 28.8\end{array}$ & $\begin{array}{c}\% 19.7 \% \\
25.6 \% 1 \\
\% 18.2 \% \\
023 \%\end{array}$ & 16.9\%19.4\% & $\begin{array}{l}19.8 \% \\
26.2 \% \\
36.5 \% \\
22.9 \%\end{array}$ \\
\hline
\end{tabular}

Fuente: Encuesta de Desarrollo Social (1997) - SIEMPRO. Procesamiento propio sobre datos de Bahía Blanca.

El análisis de la ocupación reviste una importancia peculiar. La ocupación suele ser "el" indicador por excelencia en la medición de las clases. Pero en una ciudad como Bahía Blanca, es necesario revisar el uso de dicho indicador o combinarlo con la aplicación de otros. Esto se fundamenta por los altos porcentajes de desempleo de la última década, que dejan a altos porcentaje de la población fuera del indicador u obligan a la incorporación de mano de obra secundaria (otros miembros del hogar que no son jefes de familia), o a la búsqueda de otras estrategias de supervivencia. A nivel de interacción (como se verá más adelante) las personas se ubican en determinado estrato por otras cuestiones (por ejemplo, educación), sin

\footnotetext{
${ }^{8}$ Se tomaron en cuenta los ingresos nacionales de 1997 según la EDS, por no hallarse el dato disponible según EPH para ese mismo año.
} 
asignar tanta importancia a la ocupación. Estas cuestiones simbólicas tendientes a disminuir el peso que la ocupación tiene como indicativo de clase social, se vinculan también con el hecho de que es el único indicador donde la población bahiense se encuentra en peores condiciones con respecto a los promedios nacionales. (Pérez, 2003) Sin embargo, es interesante señalar que, cuando se analiza la distribución por calificación de la ocupación, las posiciones de los bahienses mejorarían (con respecto a la categoría ocupacional) en el sentido de los otros indicadores analizados.

En la calificación ocupacional, aparece otra de las variables consideradas: la educación. Bahía Blanca presenta una amplia cobertura en el acceso a la educación primaria y altos porcentajes de personas con estudios terciarios y/o universitarios. Estos buenos niveles de educación llevan a dos situaciones: una de carácter estructural, relacionado con el tema de la ocupación, que es la sobrecalificación de la mano de obra ocupada; la otra es la sobredeterminación simbólica que dicha temática implica en el imaginario bahiense. En lo que respecta a la forma de la pirámide social, pero en este caso teniendo en cuenta la educación; y si consideramos el acceso a estudios terciarios y universitarios como el estrato superior; nos enfrentaríamos a un ensanchamiento de la cúpula de la figura piramidal, con aproximadamente el 20\% de la población mayor de 18 años (Pérez, 2003)

El patrimonio o la posesión de bienes, no muestra grandes diferencias con los promedios nacionales, aunque hay una leve diferencia en el ítem de autos a favor de Bahía Blanca. En general, la variable "cantidad de bienes" reviste una distribución normal que implicaría también (como en otros indicadores) un ensanchamiento de los sectores intermedios de la pirámide (Pérez, 2003).

Por último, y como se comentó antes con respecto a la vivienda, menos de un $3 \%$ de ellas es precaria. . Inclusive se encuentran ciertos indicadores de bienestar, como puede ser la existencia de tres o más habitaciones en el $63.64 \%$ de los hogares. Dicho bienestar también se refleja en que cerca del $85 \%$ de la población no vive hacinada. Sólo el $4 \%$ vive en hacinamiento crítico (más de 3 personas por cuarto) (Pérez, 2003).

La importancia de la vivienda en el análisis de la configuración social del espacio y su lugar privilegiado como indicador de clase social, remite a su estudio en detalle. La medición de la misma, se realiza tradicionalmente a través de algún

\footnotetext{
${ }^{9}$ EI SIEMPRO define como vivienda precaria a toda aquella que responde a la figura de ranchos, casillas, hoteles/ pensiones o inquilinatos
} 
índice, aunque otras investigaciones sintetizan toda esta información en una apreciación cualitativa proveniente de la observación por parte del encuestador. Así, según la EPH, la mayoría de los mismos viven casas (70\%), en tanto que un $26.5 \%$ lo hace en departamentos, mientras que tan solo un $1.5 \%$ habita en inquilinatos, hoteles o pensiones. La cantidad de habitaciones por vivienda varía de uno a siete, siendo la moda estadística tres, con el $37 \%$ de los casos, sólo un $12.6 \%$ dispone de una habitación y un $6.1 \%$ más de cinco (EPH- Mayo 2001).

Sautú (2001), plantea una serie de indicadores para categorizar el nivel de vivienda de la clase media de la Capital Federal. Entre ellos se menciona el porcentaje de viviendas de clase media que poseen 3 o más habitaciones. La frecuencia porcentual es igual al $73 \%$, oscilando respectivamente entre $53.9 \%, 52.5 \%$ y $95.8 \%$ en los estratos medio- bajo, medio y medio - alto. Comparada con estas cifras, que el $63.64 \%$ de viviendas totales (y no sólo de clase media) de Bahía Blanca, posea tres habitaciones o más, indica la situación favorable de la población (en lo que hace al mencionado indicador) con respecto a la Ciudad Autónoma de Buenos Aires.

En cuanto a las características de la vivienda referidas a las paredes exteriores se hallan resumidas en el siguiente cuadro.

Cuadro 3: Materiales de paredes exteriores

\begin{tabular}{|l|c|}
\hline \multicolumn{1}{|c|}{ Materiales } & Frecuencia \\
\hline Ladrillo, piedra u hormigón & $96.6 \%$ \\
Adobe & $0.1 \%$ \\
Madera & $0.3 \%$ \\
Otros & $1.0 \%$ \\
No responde & $1.9 \%$ \\
Total & 99520 \\
& \\
\hline
\end{tabular}

Fuente: Encuesta Permanente de Hogares (Mayo 2001) - INDEC. Datos de Bahía Blanca.

En cuanto al acceso al agua potable, los datos para el 2001 señalan que el $96.9 \%$ posee instalación de agua y un $1.21 \%$ no posee dicho servicio.

Como puede observarse existe un elevado porcentaje (que en algunos indicadores llega a la universalización) de personas que viven en condiciones habitacionales favorables. Tales condiciones elevan el nivel socioeconómico de las personas y homogeinizan a situaciones de clase muy dispares. Así, para señalar 
diferencias, las personas indicarán el "lugar donde está emplazado la vivienda" (barrios residenciales, "el Palihue", la zona céntrica, "lo de afuera") y no la provisión de estos servicios básicos, el hacinamiento o los materiales.

Por último, en lo que refiere a la propiedad de la vivienda, el $68.9 \%$ es propietario, el $17.5 \%$ inquilino y el $13.6 \%$ pertenece a otros regímenes. Estas condiciones favorables en la tenencia de la vivienda también se mantuvieron al combinar este indicador con otros pertenecientes a la dimensión ingresos u ocupación. Para los ingresos se trabajó con los deciles de ingreso per cápita y para ocupación, con la categoría ocupacional, ambos de la EPH 1997.

Cuadro 4: Hogares clasificados por régimen de tenencia de la vivienda (Según ingresos per capita familiar y condición de actividad- categoría ocupacional del jefe)

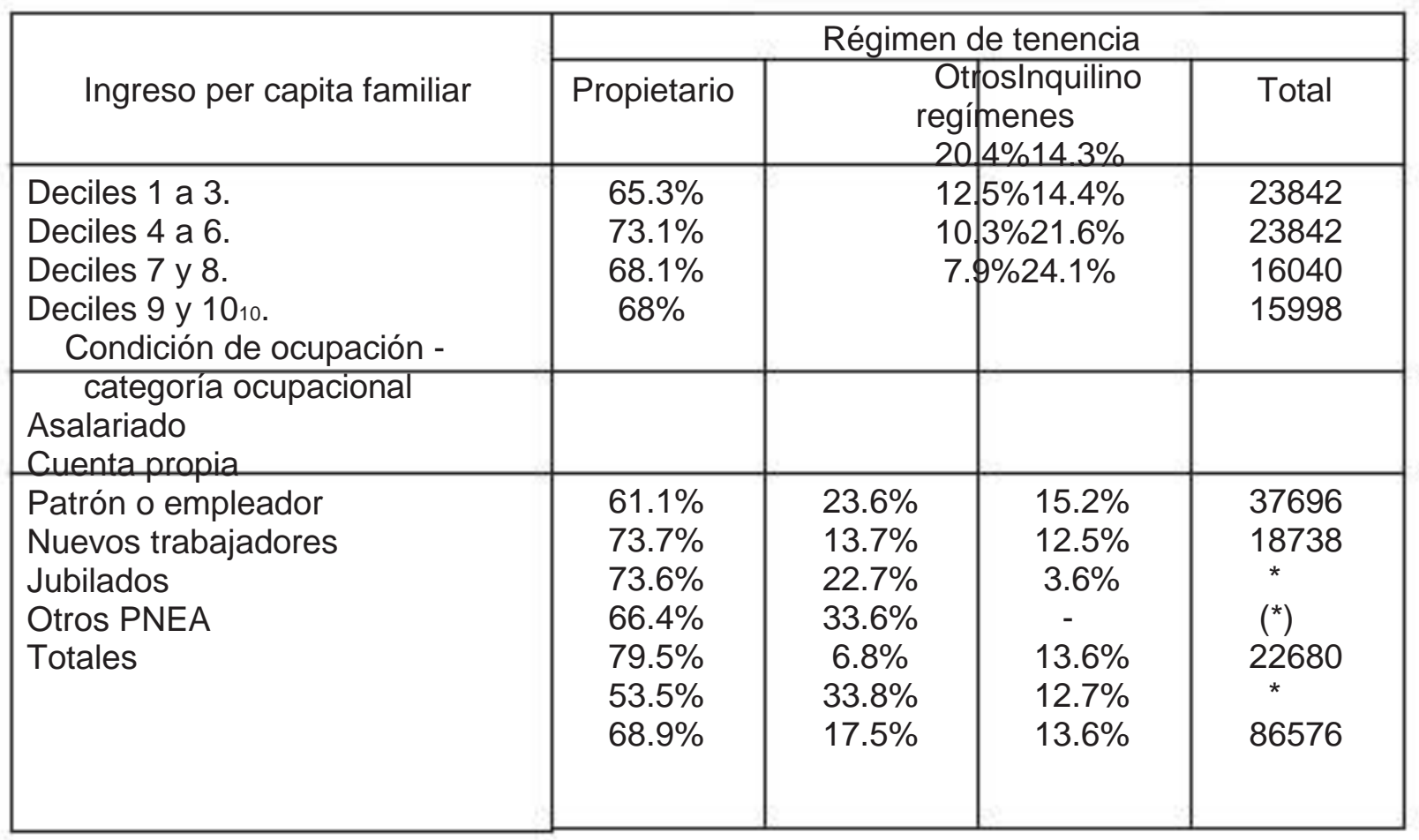

Fuente: Encuesta Permanente de Hogares (Mayo 2001) - INDEC. Datos de Bahía Blanca.

\footnotetext{
${ }^{10}$ Se eliminó de este cuadro la población que declaró no tener ingreso o desconocer el monto del mismo. La misma corresponde a 6854 personas que constituyen el $7.9 \%$ de la PEA encuestada. En la parte inferior del cuadro los asteriscos $\left(^{*}\right)$ hacen referencia a totales muy pequeños que implican probabilidades de error alto en la estimación sobre la población total. Sin embargo, estas aclaraciones no invalidan la lectura realizada del cuadro con respecto a la tenencia de la vivienda.
} 
Como puede observarse no existe mucha diferencia en las situaciones de tenencia cuando se lo controla por ocupación, y sí es un poco mayor en el caso de los ingresos. Esto refuerza lo planteado por Torrado con respecto a la situación favorable de las viviendas en las ciudades pequeñas y medianas; a la vez que indica la necesidad de revisar el lugar asignado a la variable en la determinación de la condición de la vivienda (Azuela y Tomás, 1997).

Para el año 2001, los datos presentan las mismas características en lo que hace referencia a la tenencia de la vivienda: un $67.8 \%$ es propietario de la vivienda y el terreno, mientras en 22.8 es inquilino (porcentajes que sumados representan el $90.6 \%$ de formas regulares de propiedad de la vivienda). Un 1.9\% sólo posee la tenencia regular de la vivienda (y no del terreno) y finalmente un $4.9 \%$ es ocupante de hecho o por cesión o préstamo.

\section{NUEVAS FORMAS DE DESIGUALDAD: INTERACCION SOCIAL Y DIFERENCIACION SIMBOLICA}

Los datos revisados permiten una visión estructural de la desigualdad y en especial de las condiciones habitacionales. En los análisis tradicionales de clase, estos datos suelen ser los únicos que se tienen en cuenta. Sin embargo, en la actualidad, el análisis de la desigualdad ha incorporado nuevas variables vinculadas a la cultura y la identidad, entendiéndose que un proceso tan complejo no puede limitarse a su aspecto económico, sino que también debe entender sobre las preocupaciones sociales y políticas de nuestra época.

Es necesario entonces, incorporar categorías analíticas propias de la sociología, antropología y psicología para comprender con mayor profundidad los procesos de construcción de identidad implicados en la problemática socio-política. Dentro de estas disciplinas se remite a cuerpos teóricos donde se acentúan el papel de la interacción humana y los mecanismos simbólicos, anteponiéndolos a los procesos estructurales y a los mecanismos materiales.

El estudio del uso y división del espacio urbano también ha sido influenciado por estas renovaciones. Hoy en día, no remite sólo a los factores económicos que se relacionan con el mismo, sino que se incorporan nuevos elementos no materiales y subjetivos, y se complejizan las relaciones entre ellos. Ha habido un gran cambio en las dos últimas décadas en cómo las divisiones 
espaciales/ sociales se conciben, con un giro desde las noción de división a la idea de diferencia. En parte este giro refleja el proceso de cambio social y económico, pero también da cuenta de una nueva perspectiva teórica para estudiar las ciudades (Bridges y Watson, 2003).

Esto se relaciona con cambios en la teoría sociológica contemporánea, donde hay un corrimiento de las explicaciones desde las luchas económicas a las luchas en torno a las estructuras comunicativas (Brunet y Morell, 1998). El paso de la prevalencia de una dimensión a otra obliga a la incorporación de dimensiones simbólicas propias del agente en el análisis de la ciudad y la diferenciación social.

Desde el estudio de lo urbano, Bridges y Watson (2003) señalan enfáticamente que las diferencias se construyen en, y ellas mismas construyen, la vida y los espacios de la ciudad y agregan que las desigualdades se constituyen espacialmente, socialmente y económicamente, llevando a veces a la polarización, desigualdad, zonas de fragmentación y otras veces constituyéndose en lugares de poder, resistencia y ejercicio de la identidad (Bridges y Watson, 2003).

Las diferencias no sólo se registran a nivel social, cultural o económico, sino que también se constituyen simbólicamente en grupos inscriptos en espacios y zonas con un significado particular y una práctica discursiva que puede o no ser visible a los otros. Los diferentes sujetos y subjetividades se constituyen en los espacios físicos y también en los espacios imaginados de la ciudad y las distintas relaciones de poder, se inscriben en los cuerpos urbanos. Así, no existen límites claros, ni simples divisiones entre los grupos que puedan ser fácilmente mapeados en el espacio de la ciudad., y estas complejidades raramente están representadas en las estadísticas, censos y otras fuentes cuantitativas (Bridges y Watson, 2003).

Las dificultades en el registro de este tipo de diferenciación simbólica quedan superadas al trabajar con otro tipo de información como la proveniente de entrevistas o grupos focalizados. Estas técnicas cualitativas son las pertinentes cuando la teoría subyacente otorga prioridad al agente social por sobre la estructura social. Las mismas no se orientan a la cuantificación del fenómeno sino a la caracterización detallada y profunda de sus manifestaciones específicas. En consecuencia, los resultados no pueden extrapolarse a la población, pero sirven para clarificar y orientar trabajos cuantitativos a partir de cómo determinado fenómeno social afecta el comportamiento individual.

El criterio básico en la aplicación de estas técnicas es el de promover la aparición de categorías relevantes para la descripción y explicación del problema a 
investigar En este caso específico se interrogó a personas residentes en Bahía Blanca acerca de las diferencias que perciben en la sociedad en que viven. Se tuvo especial cuidado de mantener homogeneidad en cuanto a la edad y ocupación (o la del jefe de familia para los desocupados) al interior de los grupos, pero no así con la edad, reuniendo a sujetos capaces de reproducir los discursos de los distintos segmentos de la población estudiada. Tanto las entrevistas como los grupos se registraron en cintas con excepción de dos entrevistados, donde por cuestiones técnicas se realizaron dos registros escritos para cada uno, que posteriormente se cotejaron para la elaboración del informe.

El tratamiento del material cualitativo, se hizo utilizando la técnica de análisis temático que consiste en buscar los emergentes - etiquetas, creencias, valores- que aparecen en las respuestas, para reconstruir la imagen social y la autoidentificación de las personas entrevistadas. Se trabajó a partir de la comparación entre los grupos y dentro de los mismos en la búsqueda de relaciones entre los elementos para identificar tendencias o modelos, que se apoyan en algunas ideas clave presentadas como "citas". Estos discursos se presentan respetando exactamente las palabras utilizadas por los entrevistados, a fin de reconstruir su propia perspectiva. En el caso del presente trabajo, las citas se encuentran en bastardilla en notas a pie de página.

No existieron líneas teóricas preeestablecidas para el análisis de las entrevistas, excepto la hipótesis general del interaccionismo simbólico en donde los conceptos sociales son categorías culturales construidas en las experiencias de la interacción social. En esta perspectiva, la interpretación de la experiencia es un proceso cognitivo y reflexivo que está influenciado por las circunstancias personales y sociales, sin que se limite por concepciones previas del investigador, como ocurre en los cuestionarios o entrevistas estructuradas. Este material permite entonces la interpretación de los datos numéricos sobre conductas o actitudes para los que se precisa una explicación.

Las entrevistas utilizaron como eje disparador la descripción de la ciudad de Bahía Blanca por parte de los entrevistados, con la presentación de un mapa y fotos de la misma. A partir de este disparador se les solicitó que describiesen la ciudad, los grupos que la conforman y las diferencias entre los mismos, indagándose acerca de si la descripción remitía a conceptos como clase o estrato social. En la mayoría de los casos, la descripción brindada por los entrevistados comenzaba apelando a una descripción de una "personalidad bahiense tipo", en la que se recortaba la distinción entre un "nosotros" y un "ellos", o como los plantean Bridges y Watson, un "nosotros" y un "otros". 
Las palabras "nosotros" y "ellos" sólo pueden ser entendidas juntas en su conflicto. Se entiende la pertenencia al "nosotros" sólo porque se piensa en otro grupo como el de "ellos". Los dos grupos opuestos se sedimentan, por así decir, en un mapa del mundo en el que los dos polos son antagónicos. Este antagonismo es el que hace que los grupos sean "reales" y es también el que hace verosímil la unidad y la coherencia internas que se supone poseen (Bauman, 1994). De tales caracterizaciones se puede observar cómo las diferenciaciones de los grupos, no sólo se asientan en el nivel simbólico sino que también se traducen a la lógica del espacio urbano.

Cuando se preguntaba cuáles son los comportamientos y atributos (representaciones, valores e imágenes) que hay que seguir para ser parte del grupo, se hacía referencia a cuestiones que marcan, en cierta medida, condiciones de clase media o alta y se los referencia directamente con la distribución social del espacio 11.

Algunos entrevistados asimilaron a los grupos o sectores de la ciudad de Bahía Blanca, a partir de barrios. Unánimemente se ubica al barrio Palihue, al centro del casco urbano, a la calle Alem y al barrio Patagonia, como los lugares donde reside un determinado grupo de la población y se da por entendido que en los otros lugares viven personas de menor estrato social ${ }_{12}$. El orden en que han sido mencionadas las respuestas por los entrevistados se relaciona con la frecuencia con que aparece ese barrio como delimitante de un tipo o grupo de personas 13.

\footnotetext{
$11 " . .$. ir a ciertos lugares, moverse en cierto ambiente, conocer a ciertas personas, comprar en determinados negocios, comer en ciertos restaurantes. Comprar en la Cooperativa, comer en Alem o en el de Alsina arriba, moverse en toda esa zona del Palihue" (ent. 1). En términos de una joven de 24 años: "Para mí esta dividido, por edad y otras cosas... Yo me acuerdo que hace unos meses yo lleve una amiga a Chocolate (un local bailable ubicado en el Palihue) y no es por nada, pero ella iba a Baillotage (otro local ubicado en la zona comercial céntrica), nada que ver el lugar ese..." (ent. 2).

${ }_{12}$ Palihue constituye un barrio residencial de clase alta. Se encuentra separado del resto del entramado urbano por las vías del ferrocarril y el entubado del arroyo Napostá, lo que dificulta el acceso al mismo, contando con sólo una línea de transporte público. Las otras calles que se mencionan (Alem y Alsina) pertenecen al centro de la ciudad, y se caracterizan por concentrar los comercios característicos de los sectores más altos. A pesar de no estar muy distanciados de otras zonas comerciales más populares, es también menor la cantidad de líneas públicas que transitan por ellas, llegando en el caso de Alsina, a ser nulas.

${ }_{13} \mathrm{El}$ caso de Palihue es siempre mencionado como característico de la clase alta en Bahía Blanca, aunque la mención de los otros difiere (muchas veces si el entrevistado vive en alguna de las zonas mencionadas, omite su identificación como típica de estos grupos).
} 
"Si examinamos lo que dicen los individuos de una sociedad en su expresión verbal ordinaria, descubrimos que coinciden, o casi coinciden, acerca de los criterios de valoración y que saben mucho, aunque no todo, sobre quién está más alto y quién está más bajo de acuerdo con esos criterios (Barber, 1974). La coincidencia es unánime con respecto al barrio Palihue, y refleja por un lado, un indicador económico utilizado por muchos investigadores (ubicación de la vivienda), pero también se constituye como un símbolo de alta valoración social que se aplica cotidianamente como una manifestación verbal implícita en términos a tal grado sabidos tanto por el que los usa como por el que los oye, que no necesitan hacer explícita la valoración (Barber, 1974).

La accesibilidad al precinto céntrico que se comentaba en el apartado anterior, también se constituye en criterio valorable económica y simbólicamente: la importancia del sector, tanto por su incidencia dentro de la economía de la ciudad como por su rol histórico fundacional y concentrador de actividad, posee un valor simbólico altamente significativo que no ha perdido gravitación con el paso del tiempo (Zingoni, 1998).

El tipo del lugar de residencia y su ubicación dentro de la comunidad local son también símbolos de posición de clase en todas las sociedades; ... porque la casa es el lugar donde se realizan muchas actividades importantes recreativas, de consumo, de socialización o educativas y de intimidad social (Barber, 1974). Esta cuestión se repite en distintas sociedades, aunque hay que subrayar que la existencia de barrios asociados a una clase social, no implica que todos los que habiten en esa zona pertenezcan a ese grupo social.

No sólo es el criterio residencial el que utilizan los entrevistados para identificar grupos. Otros identifican distintas pautas de consumo. Las áreas más mencionadas se vinculan con la forma de vestir, hábitos de alimentación y recreación ${ }_{14}$. En esa actitud, las diferencias en el consumo no se limitan al precio del bien, sino que se relacionan con otros aspectos como el "estilo de vida" o los "gustos"; que (como plantea Bourdieu) indican que la aversión por los estilos de vida diferentes es sin lugar a dudas, una de las barreras más fuertes entre las clases (Bourdieu, 1999).

\footnotetext{
${ }^{14}$ Un testimonio plantea: "Yo creo que hay de todo: hay grupos que sí, y otros que ... A mi me da lo mismo un pantalón de los coreanos que uno de .... No lo voy a pagar 60 , cuando son de la misma tela y todo. Pero hay gente así, careta " (entrevista 9). La palabra "careta" se utiliza en la Argentina como expresión coloquial para designar a las personas frívolas o superfluas, que pretenden simular una situación o posición que no poseen.
} 
El enfoque cultural en la teoría urbana ha desestabilizado la importancia de las explicaciones económicas de la división social y espacial, y las ha desviado a las vías en las que las diferencias se construyen a lo largo de un amplio rango de terrenos culturales y simbólicos. Para Bridges y Watson, estas adscripciones de identidad pueden representarse como poderosos espacios de resistencia y definición social (Bridges y Watson, 2003). En el caso de Bahía Blanca, la importancia asignada a la educación y a la escuela donde se estudia, se constituye en un área importante desde donde se construye (y sostiene) la identidad. Esto no quiere decir que las condiciones económicas y materiales se han vuelto insignificantes en la constitución de relaciones de exclusión y marginalidad; es más bien en el terreno de la intersección de lo económico con otros ámbitos (como el cultural y el social), donde se producen nuevas diferencias (Bridges y Watson, 2003).

Al hablar acerca de cómo se reconoce a qué clase pertenece una persona, se indica que se utiliza como criterio el máximo nivel de educación alcanzado y el prestigio de la institución a la que se asistió 15. En esto de identificar qué colegios indican pertenecer a un nivel u otro, vuelve a aparecer la combinación ingresos y educación en la figura del colegio público y privado16. También, en la distribución espacial de los colegios se verifica la concentración de los considerados de "buen nivel"académico en el centro de la ciudad, dejando en la periferia sólo algunos y en su gran mayoría de carácter público 17.

Como puede observarse, las diferencias al interior de la ciudad exceden lo meramente económico y se combinan con otras características haciendo de la ciudad un lugar lleno de heterogeneidades. Distintas marginalidades, como la raza, género, o sexualidad, u otras formas de exclusión, se interrelacionan con sitios donde se concentran desventajas de poder, y nos son simplemente una cuestión de necesidades especiales o estilos de vida, sino que están embebidas en relaciones de poder simbólicas y reales (Bridges y Watson, 2003: 257). Una ciudad que concentra los servicios educativos, comerciales, laborales y sanitarios; en el centro de la misma; excluye - y no sólo espacialmente- a los sectores radicados

\footnotetext{
15 "... no importa si está bien educado o no, sino cuántos años fue a la escuela,... a qué colegios (ent. 5)". 
en la periferia, potenciando el uso simbólico de la ubicación espacial como distintiva de clase.

Las fuerzas que producen ciudades fragmentadas y divididas son múltiples. En el mercado de la tierra, la acumulación de beneficios opera como una fuerza económica crucial en la forma de la ciudad. (Bridges y Watson, 2003). Sin negar la importancia de esta fuerza, es importante señalar otras como la expansión de las clases medias y sus preferencias en el consumo18. En Bahía Blanca (y en la Argentina en general), las tendencia de la amplia mayoría de las personas a ubicarse en sectores intermedios de la escala social, sea cual sea su posición objetiva. La pregunta que se utilice para medir (Sautú, 2001), condiciona el uso del espacio urbano al inclinar las preferencias y hacer valioso un "lugar", cuando este último responde a los patrones de conducta de los sectores medios. Dicho de otra manera, un barrio o vivienda que responda a criterios de consumo típicos de clase media, se constituye en valiosa para sectores que no son objetivamente pertenecientes a dicho estrato social.

Existen otros dos aspectos interesantes en el texto ya mencionado de Bridges y Watson con respecto a diferencias urbanas no económicas. Uno es el referido a un trabajo allí citado de Brendan Gleeson, quien presenta el caso de ciertas ciudades deshabilitadoras en su diseño, en el sentido de que el desarrollo físico de la ciudadtanto de las formas de uso de la tierra como el diseño interno de los edificiosdiscriminan gente con discapacidades por no reconocer sus requerimientos de movilidad (Bridges y Watson, 2003). Para el caso de Bahía Blanca, la existencia de semáforos para ciegos, veredas con pendientes para el uso de sillas de ruedas, edificios con rampas de acceso e institutos especiales se reduce al centro urbano donde se concentra la actividad económica y política. De ahí que la ciudad - con excepción de la zona mencionada- se transforme en deshabilitadora, siguiendo la terminología acuñada por Gleeson, para las personas con dificultades físicas, más allá de su nivel socio- económico.

El segundo hace referencia al "rescate de la noción de ciudadanía en el terreno de la diferencia" (Bridges y Watson, 2003). El análisis de las entrevistas realizadas en la ciudad de Bahía Blanca, indican que dentro de la noción de ciudadanía, algunas de las creencias políticas no parecen sufrir modificaciones según clase social. Ejemplos de éstas son la percepción de los políticos, la idea de

\footnotetext{
${ }^{18}$ Bridges y Watson (2003: 257) hacen referencia al trabajo de Chris Hamnett (capítulo 28), quien asocia estos cambios a los acontecidos en la estructura social.
} 
la no existencia de fuertes ideologías, la percepción acertada sobre motivaciones políticas de otros grupos; y la definición del futuro político de Argentina como incierto. Así, otra serie de diferencias al interior de la ciudad posee una raíz no económica, y a su vez, también influye y se traduce en usos diferenciados del espacio público. Un ejemplo de esto, es el conflicto sobre la gesión del Teatro y el Ballet Municipal, donde las distintas concepciones sobre lo que es y no es cultura se traducen en modificaciones de las carteleras, los lugares donde se desarrollan los espectáculos, el público a los que se los destina, sin encontrarse una relación determinada con el nivel socio- económico, sino más bien con cierta ideología partidaria local.

Dentro de aspectos más generales de la idea de ciudadanía como diferencia se observó que es la autoidentificación de clase, y no la posición objetiva en el espacio económico- social, la que define cierta creencia política. Así, las diferencias ciudadanas con otros, se recortan desde un plano subjetivo, donde no es tan importante cuánto tengo (o tiene el otro) sino más bien, cuánto y qué creo tener. Mi relación con otros, mi identificación con un grupo político, y mi definición como ciudadano y habitante de la ciudad, se construye más a partir de la autoidentificación de clase, que de la definición objetiva de la misma a través de los indicadores económicos.

Combinando el planteo de Bridges y Watson sobre la expansión de las clases medias y sus ideas acerca de las diferencias desde la ciudadanía se observa en las entrevistas que las extendidas condiciones de clase media percibidas por los propios bahienses retroalimentan la difusión de ideas políticas vinculadas a dicho sector social. Así, la eficiencia, la moderación y la presencia activa y de gestión del Estado municipal, son elementos altamente mencionados por quienes se autoperciben en sectores medios y altos; y la ausencia de mención se hace notable entre quienes se perciben fuera (o en la base) del sistema.

En el mismo artículo, Bridges y Watson (2003) presentan también al trabajo de David Sibley (1995) donde se examinan los aspectos más simbólicos de la exclusión y de la división espacial donde la gente es marginalizada, porque son temidos y definidos como "otros". En el mismo sentido, para el caso de la provincia de Buenos Aires, el Informe para el Desarrollo Humano presentado por la Fundación del Banco Provincia de Buenos Aires (2004-2005) se plantea que la ciudad sólo es excepcionalmente un espacio común, privando no solamente a todos de la posibilidad de circular libremente por las calles y lugares públicos, sino también minando la conciencia y responsabilidad de formar parte de una comunidad integrada, pagando con "miedo" el costo de que se pierdan los atributos de la ciudadanía (Fundación 
BAPRO, 2005). Se incrementa el desconocimiento por lo extraño, cuando no directamente el rechazo. En algunos casos, ha pasado a ser diferente aún el evidentemente similar, ya que el desconocimiento y el desinterés en el otro hacen que parezca mucho más extraño de lo que en realidad es (Fundación BAPRO, 2005). En una ciudad como Bahía Blanca, donde los indicadores de ingreso muestran una mejor distribución que la observada a nivel provincial y nacional, el proceso de "extrañamiento" se construye sobre otros indicadores: el barrio en que se reside y la educación. Por otro lado, también debe reconocerse que los niveles de violencia y desintegración son menores a los que se observa en otras ciudades del país, aunque dan lugar a observaciones como la de Zingoni (1998) en cuanto a que es la población de los asentamientos quien paga los mayores costos de una mala fama y una imagen negativa generalizada, contribuyendo a afirmar la condición de marginalidad en la que viven. Si consideramos a la ciudad como un espacio múltiple de integración, no deben ser dejados de lados estos aspectos culturales y/ o simbólicos en los análisis del espacio urbano.

Estas son algunas formas de diferenciación no económicas al interior de las ciudades. Como se comentó con anterioridad, su importancia es creciente y su registro dificultoso: los mapeos estadísticos inevitablemente ignoran dichas formas recónditas de identificación y construcción de identidades en los espacios de la ciudad. El sujeto posmoderno es uno descentrado, el cual está parcialmente formado y constituido en sitios y discursos diferentes en diferentes momentos para diferentes propósitos estratégicos. La ciudad es un lugar clave en esto (Bridges y Watson, 2003).

\section{REFLEXIONES FINALES}

El presente trabajo intentó presentar la problemática urbana de Bahía Blanca vinculada al estudio de la desigualdad y la pobreza. La perspectiva urbana permite el análisis de la ciudad en forma holística e integral, integrando múltiples y diversas variables así como las complejas relaciones entre las mismas.

El estudio de lo urbano, lleva en la actualidad a la aparición de "interesantes análisis que incluyen aspectos culturales y psicosociales, los cuales enriquecen indudablemente los estudios acerca de este importante tema de la investigación" (Schteingart, 2001). Así se ha dado preeminencia al texto de Bridges y Watson, donde se resalta la importancia de distintas formas de diferenciación al interior de la 
ciudad, que también influyen en la construcción del espacio urbano y en la identidad de quienes habitan los mismos. Como mencionan los autores: las diferencias y las identidades en las ciudades se constituyen en formas múltiples y complejas, en múltiples espacios de la ciudad, y giran y cambian produciendo espacios diferentes y nuevos límites y bordes en la ciudad (Bridges y Watson, 2003).

Sin embargo, se considero necesario incluir el análisis de la dimensión urbana y de las desigualdades objetivas (ingreso, ocupación, etc) porque dan marco y permiten una comprensión más acabada de las desigualdades "no económicas". Un espacio urbano, relativamente organizado; la inexistencia de importantes problemas habitacionales, la homogeinización de los niveles de ingreso, las altas tasas de desocupación que llevan a la restructuración del mercado de trabajo y la expansión de criterios simbólicos de distinción y diferenciación característicos de los sectores medios revisten a la ciudad de ciertas particularidades. Por otro lado es importante señalar que la misma, no escapa a la lógica de las localidades pequeñas, las cuales están en mejores condiciones relativas que las de igual posición social en aglomeraciones más grandes (Torrado, 1992).

El análisis de la problemática de la vivienda, provee de otro importante puente entre la problemática urbana y la de la pobreza. Aunque este trabajo no presenta un mapa social como resultado final, la presentación de los datos habitacionales junto a otras formas de diferenciación, constituye desde la perspectiva de la sociología urbana, un avance en la comprensión en la relación entre las divisiones sociales y espaciales en la ciudad de Bahía Blanca, señalando que los diferentes espacios de la ciudad constituyen identidades y posibilidades diferentes dentro de una red de conexiones global/ local, las cuales hacen único todo lugar cualquiera sea la forma general que pueda tener (Bridges y Watson, 2003).

\section{REFERENCIAS BIBLIOGRAFICAS}

Azuela, A. y Francois, T., (coords.) (1997), El acceso de los pobres al suelo urbano. Universidad Nacional Autónoma de México. Centro de Estudios Mexicanos y Centroamericanos, México.

Bauman, Z. (1994), Pensando sociológicamente. Ediciones Nueva Visión. Buenos Aires.

Bourdieu, P. (1999), La distinción. Criterio y bases sociales del gusto. Editorial Taurus. Madrid. Primera edición en francés: 1979. 
Bridge, G. y Watson, S. (2003):, "City Differences". En Bridge, G. y Watson, S. (eds.): A Companion to the City. Blackwell Publishing, Oxford, Inglaterra.

Brunet, I. y Morell, A.,(1998), Clases, educación y trabajo. Editorial Trotta, Madrid. Burstein, N. y Pérez, S. (2004), Programa de conocimiento de las necesidades de vivienda del partido de Bahía Blanca. Documento de Trabajo del Instituto de Economía. Universidad Nacional del Sur. Bahía Blanca.

Formiga, N. y Garriz, E. (1998), "Empleo y marginalidad en la ciudad de Bahía Blanca". En M. Cernadas de Bulnes y Bustos Cara, R.(comp.) Estudios Regionales Interdisciplinarios. EDIUNS, Bahía Blanca.

Fundación Banco Provincia de Buenos Aires (2005): Integración Social de la Juventud. (Informe sobre Desarrollo Humano 2004-2005). Buenos Aires.

Prevot- Schapira, M (2001), "Fragmentación espacial y social: conceptos y realidades". En Perfiles Latinoamericanos. Número 19, Diciembre 2001.

Sautú, R. (2001), La gente sabe. Interpretaciones de la clase media acerca de la libertad, la igualdad, el éxito y la justicia. Editorial Lumiere. Buenos Aires.

Schteingart, M. (2001), "La división del espacio social en las ciudades". En Perfiles Latinoamericanos. Facultad Latinoamericana de Ciencias Sociales, México. Año 9, número 19, diciembre. México.

Schteingart, M. y Duhau, E. (coords.) (2001), Transición política y democracia municipal en México y Colombia. GURI, Miguel Ángel Porrua Editores, Colección las Ciencias Sociales, México.

Torrado, S. (1992), Estructura social en la Argentina.1945-1986. Colecciones del Trébol. Buenos Aires.

Zingoni, J. (1998), Plan Estratégico de Bahía Blanca. Documento de pre- diagnóstico. Síntesis. Bahía Blanca. 